10 years ESJ

Special edition

\title{
El arte en la promoción de la educación intercultural en contextos escolares
}

\author{
Jenny Sousa, Doctorada en Estudios Culturales \\ Escola Superior de Educação e Ciências Sociais, CICS.NOVA.IPLeiria- \\ iACT, CI\&DEI, Politécnico de Leiria, Portugal
}

Doi: 10.19044/esj.2021.v17n26p13

Submitted: 22 March 2021

Accepted: 18 May 2021

Published: 09 August 2021
Copyright 2021 Author(s)

Under Creative Commons BY-NC-ND

4.0 OPEN ACCESS

Cite As:

Sousa J. (2021). El arte en la promoción de la educación intercultural en contextos escolares. European Scientific Journal, ESJ, 17 (26), 13. https://doi.org/10.19044/esj.2021.v17n26p13

\section{Resumen}

Las sociedades contemporáneas son más que lugares de diversidad cultural y multiculturalismo; son espacios que buscan la convivencia armónica de sus ciudadanos, en una sana interacción de diferentes culturas y construcción de expresiones culturales compartidas a través del diálogo y el respeto mutuo. En otras palabras, las sociedades actuales, en su modo de existencia, buscan la interculturalidad. En todo este proceso, la educación intercultural se destaca como parte integral de la educación para la ciudadanía y para los valores y principios de los derechos humanos. Ante esta situación, la Escuela está llamada a jugar un papel preponderante, ya que constituye un espacio privilegiado para prácticas de aprendizaje y perspectivas del enfoque intercultural. En este sentido, las artes emergen como herramientas competentes en la construcción de estos procesos educativos, promoviendo la acción ciudadana de todos los involucrados, a través de metodologías pedagógicas más acordes con las relaciones educativas interculturales. Para construir contextos escolares cada vez más interculturales e inclusivos, surge en Portugal el Plan Nacional de las Artes, que reitera la importancia de las artes en la promoción de prácticas educativas más igualitarias, ricas e innovadoras. En vista de lo anterior, en este trabajo se realiza una reflexión teórica que, cruzada con un análisis de carácter más práctico, nos permite darnos cuenta de que las artes son parte central de la experiencia humana y 
que, como tales, constituyen oportunidades para la construcción de procesos de interculturalidad y educación intercultural, es decir, en contextos escolares.

Palabras clave: Interculturalidad, educación intercultural, escuela contemporánea, arte, Plan Nacional de las Artes

\title{
Art in Promoting Intercultural Education in School Contexts
}

\author{
Jenny Sousa, Doctorada en Estudios Culturales \\ Escola Superior de Educação e Ciências Sociais, CICS.NOVA.IPLeiria- \\ iACT, CI\&DEI, Politécnico de Leiria, Portugal
}

\begin{abstract}
Contemporary societies are more than places of cultural diversity and multiculturalism; they are spaces that seek the harmonious coexistence of their citizens, in a healthy interaction of different cultures and the construction of shared cultural expressions through dialogue and mutual respect. In other words, today's societies, in their mode of existence, seek interculturality. In this whole process, intercultural education stands out as an integral part of education for citizenship and for the values and principles of human rights. In view of this situation, the School is called to play a predominant role, since it constitutes a privileged space for learning practices and perspectives of the intercultural approach. In this regard, the arts emerge as proficient tools in the construction of these educational processes, promoting the citizen action of all those involved, through pedagogical methodologies that are more in line with intercultural educational relationships. To build increasingly intercultural and inclusive school contexts, the National Plan of Arts emerges in Portugal, which reiterates the importance of the arts in promoting more egalitarian, rich and innovative educational practices. In view of the above, a theoretical reflection is carried out in this work, which, crossed with an analysis of a more practical nature, allows us to realize that the arts are a central part of the human experience and that, as such, constitute opportunities for the construction of interculturality and intercultural education processes, namely in school contexts.
\end{abstract}

Keywords: Interculturalidad, educación intercultural, escuela contemporánea, arte, Plan Nacional de las Artes 


\section{Introduction}

Pensar la sociedad en los días de hoy implica tener en línea de cuenta la diversidad cultural, en una lógica que supera al multiculturalismo y encuentra eco en la esencia intercultural. De hecho, las sociedades con características interculturales no solo reconocen la existencia, en su corazón, de diferentes culturas, sino que comprenden esta diversidad como fuente de riqueza y una mejor comprensión y renovación de identidades (Candau, 2008; Giménez, 2010; Silva \& Rebolo, 2017).

En todo este proceso, la educación intercultural se destaca como parte integral de la educación para la ciudadanía y los valores y principios de los derechos humanos. En este contexto, la Escuela es llamada a tener un papel de liderazgo, ya que constituye un espacio privilegiado para el aprendizaje compartido de prácticas y perspectivas del enfoque intercultural. Así, la educación intercultural, que se materializa principalmente a través de la mano del Docente Intercultural, es mucho más que una asignatura académica que se imparte; más bien, es la creación de procesos de experiencias colectivas, en las que toda la comunidad educativa se desarrolla y aprende, a partir de lo que observa e interactúa.

En este sentido, las artes y los lenguajes artísticos cobran especial importancia en estos procesos educativos, promoviendo la acción ciudadana de todos los involucrados, a través de metodologías pedagógicas que potencien el aprendizaje de todos, originando simultáneamente el éxito y el bienestar de niños, jóvenes y adultos, en una nueva cultura con mayor permeabilidad otorgando mayor riqueza (Almeida, 2010; Silva \& Rebolo, 2017; Sousa, Lopes \& Kowalski, 2020).

En el trabajo que sigue, empezamos por reflexionar sobre los conceptos de diversidad cultural, multiculturalidad e interculturalidad, señalando los puntos de similitud, pero, sobre todo, las posiciones divergentes, canalizando la discusión a la importancia de pensar interculturalmente.

A continuación, se discute la importancia de la Escuela contemporánea como espacio primordial para la promoción de la igualdad de oportunidades y la integración de diferentes personas, en un enfoque basado en los principios de la educación intercultural.

En el tercer punto, se analiza el potencial de las artes y de los lenguajes artísticos como herramientas pedagógicas para cambiar la escuela, su organización y enfoque, apuntando a la interculturalidad. En este apartado, también se analiza el Plan Nacional de las Artes, plan estratégico recientemente implementado en Portugal, y se reflexiona sobre su papel y contribución en el contexto de la educación intercultural desarrollada en este país.

Así, y en vista de lo anterior, este trabajo pretende realizar una reflexión teórica que, cruzada con un análisis de carácter más práctico, 
estimule el debate y aumente el conocimiento sobre la importancia de las artes en la promoción de la educación intercultural en los contextos escolares.

\section{Diversidad cultural e interculturalidad:}

A mediados del siglo XXI, el concepto de interculturalidad ha cobrado importancia renovada en una sociedad que quiere ser igualitaria, democrática y en convivencia con los derechos humanos. Independientemente del grado de pluralismo cultural que caracteriza a la comunidad, ahora se aboga por un cambio de paradigma, pasando del multiculturalismo a la interculturalidad. En relación a este tema, Antônio Pierucci aclara que "parece generalizarse, a un ritmo acelerado y perturbador, la conciencia de que los humanos somos realmente diferentes [...], pero también somos diferentes en derecho" (1999, p. 7); ahora, en su opinión, "ya no queremos la igualdad, parece, o la queremos menos, nos motiva mucho más, en nuestra conducta, en nuestras expectativas de futuro y en los proyectos de vida compartidos, el derecho a ser personal y colectivamente diferentes de los demás" (Pierucci, 1999, p. 7). Y en su argumento, el autor deja las siguientes preguntas: " $¿$ Somos todos iguales o somos todos diferentes? ¿Queremos ser iguales o queremos ser diferentes?" Estas preguntas parecen encontrar una respuesta, algunos años después, en palabras de Boaventura Sousa Santos:

Tenemos derecho a ser iguales cuando nuestra diferencia nos hace inferiores; y tenemos derecho a ser distintos cuando nuestra igualdad nos desnaturalice. Así que resulta, la necesidad de una igualdad que reconozca las diferencias y una diferencia que no produzca, alimente o reproduzca desigualdades. (Santos, 2003, p. 56)

La interculturalidad, por lo tanto, parece ser la forma correcta de construir sociedades que reconozcan y respeten las diferencias. De hecho, al ser una nueva expresión dentro del pluralismo cultural, la interculturalidad afirma lo diferente y lo común, a través de la promoción de prácticas que generan igualdad, libertad e interacción positiva en la relación entre individuos, grupos y comunidades culturalmente distintas (Giménez, 2010).

En este contexto, el telón de fondo es la diversidad cultural, elemento que caracteriza cada vez más a la sociedad actual (diversos orígenes étnicos, lenguas y tradiciones culturales). En realidad, para que la convivencia entre personas sea armónica, es necesario promover valores como la integración, la tolerancia, el respeto mutuo y la inclusión. Por ello, la interculturalidad se destaca como un proceso de aceptación y normalización de las diferencias culturales, concentrándose en las cuestiones que más interesan.

Según UNESCO (2005), la interculturalidad se refiere a la existencia e interacción equitativa de diferentes culturas, así como a la posibilidad de 
generar expresiones culturales compartidas a través del diálogo y el respeto mutuo.

Así, y en línea con lo presentado hasta ahora, hablar de interculturalidad es ir más allá de la multiculturalidad. Aunque estos dos conceptos tengan similitudes, lo que significa que muchas veces se entienden como sinónimos, lo cierto es que estos conceptos también tienen muchas diferencias. Para la UNESCO (2006), el término multicultural describe la naturaleza culturalmente diversa de la sociedad humana. No solo se refiere a elementos de la cultura étnica o nacional, sino que también incluye la diversidad lingüística, religiosa y socioeconómica. A pesar de defender la igualdad de derechos y oportunidades, plasmado en el derecho a la diferencia de experiencias culturales, este enfoque se centra demasiado en los contrastes culturales, entendiendo la cultura como cerrada en sí misma, cristalizada y uniforme (Giménez, 2010).

En esta reflexión ya surge una idea notable: la multiculturalidad expresa las diferencias como un hecho objetivo, mientras que la interculturalidad aspira a que estas diferencias se traduzcan en la plena integración de las culturas. De esta manera, la interculturalidad tiene un carácter reivindicativo y transformador, ya que propone superar la multiculturalidad, como se lee en el documento "Términos de Referencia", de la Red de Escuelas de Educación Intercultural (REEI, 2017).

Por consiguiente, se pretende un paradigma que crea un marco de convivencia en el que ningún grupo es discriminado ni excluido, por los aspectos diferenciadores. Así, las diferencias entre un grupo y otro no pueden involucrar ninguna forma de desigualdad, contradiciendo el legado dejado por la sociedad tradicional, que estableció jerarquías basadas en aspectos diferenciadores. Este nuevo paradigma, que se materializa en la interculturalidad, se basa en un criterio ético: todas las culturas merecen el mismo respeto.

La interculturalidad rompe con la visión esencialista de las identidades culturales y concibe las culturas en un proceso continuo de elaboración, construcción y reconstrucción. Ciertamente, cada cultura tiene sus raíces, pero estas raíces son históricas y dinámicas. No imponen a las personas estándares culturales. Sin embargo, y como advierte Candau, es necesario ser consciente de que "las relaciones culturales no son relaciones idílicas, no son relaciones románticas; están construidas en la historia y, o sea, están atravesadas por cuestiones de poder, por relaciones fuertemente jerárquicas, marcadas por el prejuicio y la discriminación de ciertos grupos" (2008, p. 51).

Sin embargo, prosigue el autor, "la perspectiva intercultural afirma esta relación, que es compleja y admite configuraciones distintas en cada realidad, sin reducir un polo al otro" (Candau, 2008, p. 51). Este es el 
verdadero reto de la interculturalidad, que se materializa en la promoción de una educación para el reconocimiento del "otro", plasmada en el diálogo entre los diferentes grupos sociales y culturales:

Una educación para la negociación cultural, que tilde los conflictos provocados por la asimetría de poder entre los diferentes grupos socioculturales de nuestras sociedades y sea capaz de favorecer la construcción de un proyecto común, en el que las diferencias se integren dialécticamente. La perspectiva intercultural se orienta a la construcción de una sociedad democrática, plural, humana, que articule políticas de igualdad con políticas de identidade. (Candau, 2008, p. 52)

Ahora, como se explicó, en esta ecuación se destaca la educación intercultural como vehículo privilegiado en la construcción de una sociedad más plural y humana. De esta ecuación también surge otro elemento: la Escuela.

Los contextos educativos del sistema educativo, es decir, las escuelas, se asumen como parte integrante de la Comunidad en la que se insertan y acuden a ella simultáneamente. Por eso, además de reconocer la multiculturalidad, la escuela actual percibe los retos de la interculturalidad y ve la educación intercultural como una herramienta importante en la construcción social de comunidades más humanas. La escuela queda así demarcada como un contexto vivencial donde la interculturalidad es un beneficio de y para todos.

\section{La educación intercultural en la escuela contemporánea}

Como ya se comentó en el punto anterior de este trabajo, el pluralismo cultural es una característica cada vez más preponderante de las sociedades contemporáneas, donde Portugal no es una excepción.

El sistema educativo portugués es consciente de la importancia de reconocer y valorar la creciente diversidad cultural y lingüística, no solo de los estudiantes, sino de toda la comunidad educativa. Esta diversidad, que va mucho más allá del origen político geográfico o de los idiomas hablados por los sujetos, constituye una oportunidad para mirar lo distinto, comprenderlo y apoyarse en él (REEI, 2017).

Esta riqueza también puede reflejarse en el dominio del desarrollo curricular, insertando los aportes que la participación activa de cada elemento favorece en el proceso de enseñanza-aprendizaje, al tiempo que potencia la diferenciación pedagógica.

Se recomienda, sobre todo, posibilitar la igualdad de oportunidades y la integración de diferentes personas en la escuela y, principalmente, en la 
sociedad. Si bien las escuelas no cambian la sociedad por sí solas, no debemos olvidar que estos son espacios privilegiados para construir focos de resistencia, que brindan modelos pedagógicos para nuevas formas de aprendizaje y relaciones sociales.

De este modo, el objetivo es construir una nueva cultura organizacional, basada en los éxitos y el bienestar de todos los involucrados, basada en las prácticas escolares y la producción de obras en perspectivas que abordan la diversidad cultural (Silva \& Rebolo, 2017). En rigor, se trata de incorporar la educación intercultural como elemento estructurante.

La educación intercultural presupone una perspectiva dinámica y un conjunto de dimensiones transversales, en el ámbito del desarrollo personal y social, basado en la comunicación abierta y la cooperación entre socios (Cornejo, 2012).

Es un proceso continuo y, como tal, no puede reducirse a unas situaciones concretas de realización de actividades concretas, ni puede centrar la atención en determinados grupos sociales (Silva \& Rebolo, 2017). En efecto, la educación intercultural tiene una perspectiva global y afecta a toda la comunidad escolar y a todos los agentes involucrados en los procesos educativos (Almeida, 2010; Cornejo, 2012; Sousa, Lopes \& Kowalski, 2020).

Por ello, la Escuela reconoce y valora la diversidad y el pluralismo cultural "como riquezas para promover una educación inclusiva que lleve al desarrollo de la identidad de cada uno, al diálogo, a la interacción, al encuentro con el otro, al análisis constructivo de la diversidad y al éxito educativo" (REEI, 2017).

En la Escuela que considere la educación intercultural como un proceso educativo bajo su responsabilidad, el papel del profesorado es central, ya que la implementación efectiva del principio de igualdad de oportunidades para el éxito en la educación escolar es intrínsecamente dependiente de las actitudes y comportamientos interculturales / multiculturales que adopta (Almeida, 2010).

A menudo, explican Stoer y Cortesão (1999), los docentes no son sensibles al "arco iris" de culturas que tienen ante sí y tienden a ignorar la importancia de adaptar el currículo con el que trabajan o la relación pedagógica que establecen. Aún así, según los autores, en la mayoría de los casos, los profesores perciben la diversidad como un problema y no como un recurso. Al mismo tiempo, tienden a comprender esta diversidad desde un punto de vista individualista, especialmente considerando aspectos como la motivación, la personalidad o las habilidades, ignorando factores contextuales (Stoer \& Cortesão, 1999).

La Escuela Intercultural debe tener un Docente Intercultural. Los docentes son los principales mediadores entre las culturas, la de la escuela y la de los estudiantes, y están posicionados en una interfaz entre el estudiante, 
la escuela y la familia, y pueden contribuir significativamente a la facilitación de los procesos de construcción intercultural (Almeida, 2010).

En este objetivo, para promover la igualdad y la integración de los diferentes sujetos socioculturales en la escuela y la sociedad, "el docente debe posibilitar dinámicas del proceso de enseñanza y aprendizaje que integren conocimientos diversos y que posibiliten conocimientos enriquecidos por la diversidad" (Branco, 2021, p. 314).

La Escuela Intercultural se basa en la educación intercultural que no se limita al aula y reconoce que las prácticas pedagógicas ricas e innovadoras deben ser, a la vez, interculturales, es decir, "la Educación Intercultural se vive como un proceso continuo, en permanente adaptación al contexto, [que] pasa por toda la praxis de quienes trabajan y viven en ella" (REEI, 2017).

En este ámbito, las artes tienen un gran potencial como herramientas pedagógicas para transformar la escuela, su organización y enfoque, apuntando a la interculturalidad.

\section{Las artes en la educación intercultural: una forma de operacionalización}

El arte y la educación son capaces de promover relaciones e interacciones críticas y creativas, desencadenando procesos de conocimiento y convivencia intercultural. Hay que tener en cuenta que en los procesos artísticos se movilizan diversos campos de acción, conceptos, interacciones y experimentos que enriquecen los movimientos interculturales.

Las artes forman parte de la experiencia humana y, al mismo tiempo, permiten la autoexpresión, promueven el conocimiento del otro y de la realidad externa (Sousa, Lopes \& Kowalski, 2020). A través del arte, el mundo se vuelve más inteligible, asequible y familiar y se revela como una "fuerza poderosa y omnipresente que ayuda al individuo a moldear actitudes, creencias y comportamientos. En este sentido, el arte es, ante todo, una expresión de la existencia en la sociedad y un instrumento para comprender las actitudes de la vida" (Sousa, 2020).

Ahora bien, con base en este argumento, no es de admirar que las artes se destaquen como herramientas privilegiadas en la construcción de escuelas que quieran ser interculturales e inclusivas.

Las artes, y el fomento de la experimentación artística, son vehículos fundamentales en la promoción de la participación activa, en el itinerario del aprendizaje, de todos los niños y jóvenes, valorando su patrimonio cultural y origen nacional, condición jurídica y situación socioeconómica (REEI, 2017).

Dado que el arte vive en una relación profunda con la comunidad, "la contribución social de las artes se entiende predominantemente como una contribución formativa y empoderadora en sí misma. Tales competencias son decisivas para la formación de las personas y su capacidad para superar barreras sociales, económicas y simbólicas" (Fortuna, 2014). Así, pensar en 
una educación intercultural basada en el arte, es transformar los procesos educativos en vehículos de experimentación y participación en la comunidad, adaptando metodologías, entendiendo e inventando propuestas en un contexto educativo: "se trata de vivir experiencias y aprender con experiencias únicas y procesos cambiadores y por eso se genera aprendizaje" (Sousa, 2020, p. 8).

En las relaciones educativas interculturales, el arte puede alentar a los diversos elementos de la comunidad educativa a ver significados, reflexionar y construir significados. Al crear espacios de experimentación artística, las prácticas educativas pueden desencadenar procesos de aprendizaje problemáticos y reflexivos, basados en la articulación de sentidos y significados producidos en el ámbito de la diversidad cultural.

En vista de lo anterior, el uso del arte, independientemente del lenguaje artístico, tiene la capacidad de producir diferentes posibilidades educativas, en el ámbito de la educación intercultural. En este apartado, "el gran reto es involucrar a los estudiantes en el proceso de enseñanza y aprendizaje, promoviendo el relevamiento de conocimientos previos, con el fin de identificar sus conocimientos y tenerlos como punto de partida para el trabajo a realizar" (Branco, 2021).

Así, y en base a lo explicado hasta ahora, ¿puede el Plan Nacional de las Artes (2019), en adelante PNA, convertirse en una herramienta para impulsar procesos de educación intercultural?

Con la asunción del artículo 78 de la Constitución portuguesa - Fruto y creación cultural - la PNA fue instituida por el Ministerio de Cultura y el Ministerio de Educación, para el horizonte temporal 2019-29, teniendo en cuenta "la necesidad de organizar, promover e implementar, de manera articulada, la oferta cultural para la comunidad educativa y para todos los ciudadanos, en una lógica de aprendizaje permanente" (PNA, 2019, p.10). Entre los diversos objetivos planteados en el documento, se considera especialmente pertinente, en el ámbito de este trabajo, plantear los siguientes: “e) Fomentar la colaboración entre artistas, educadores, docentes y estudiantes, con el fin de diseñar estrategias de enseñanza y aprendizaje. que promuevan un currículo integrador, basado en una gestión consolidada del conocimiento y la experiencia cultural" y "k) Promover el conocimiento, la integración y el encuentro de culturas, a través de las manifestaciones artísticas y culturales de diferentes comunidades" (PNA, 2019, p.10).

Es, por tanto, una estrategia que reclama viejas relaciones, pero que también provoca nuevas conexiones en el ámbito del arte y la educación:

"El arte es un lenguaje universal, que transmite significados imposibles a cualquier otro tipo de lenguaje, quiere sea semántico, dialógico o científico. Así, educar para la ciudadanía, para la transformación social, para el bienestar colectivo, es imposible si la educación no engloba la dimensión artística y patrimonial”. (PNA, 2019, p. 11) 
Se invita a la Escuela y la Comunidad a desarrollar una articulación fructífera, basada en el poder educativo, transdisciplinario e indisciplinado de las Artes. La importancia del papel de las artes en la educación, particularmente a nivel de educación intercultural, no es nueva en Portugal, y ya se reflejó en el documento sobre el Perfil de los estudiantes al final de la escolaridad obligatoria, pero parece cobrar un nuevo impulso con el Plan Nacional de las Artes, plan estratégico para el acercamiento y el arraigo de las artes en las escuelas, teniendo en cuenta la coincidencia entre los temas de Ciudadanía y Desarrollo y los que aparecen en las manifestaciones artísticas de todos los tiempos.

Así, el Plan Nacional de las Artes (2019) asume como Misión promover, en territorio portugués, con ciudadanos de todas las edades, en particular niños y jóvenes, la "transformación social, movilizando el poder educativo de las artes y el patrimonio en vida de ciudadanos: para todos y con cada uno" (p. 14).

El PNA forma parte de una estrategia concertada para colaborar en la creación de políticas integradas (cultura y educación), para acercar el arte a la ciudadanía, especialmente a niños y jóvenes, para estimular la difusión y preservación del patrimonio histórico (material e inmaterial); fomentar la circulación de la creación contemporánea y, por consiguiente, trabaja en conjunto con el Plan Nacional de Lectura (PNL2027), la Red de Bibliotecas Escolares, el Plan Nacional de Cine, el Programa de Educación Artística y Estética, la Red de Museos Portugueses, con el fin de articular y potenciar la acción de estos programas y redes.

La puesta en funcionamiento de este Plan se realiza a través de un plan de acción estratégico, que se sustenta en tres ejes principales: Eje A - Política cultural, Eje B - Formación y Eje C - Educación y Acceso. Cada uno de estos ejes está compuesto por Programas, estando cada uno de estos Programas compuesto por Medidas. Si bien todos los ejes son importantes en el contexto de la temática abordada en este artículo, es en el Eje C - Educación y Acceso, donde hay una mayor aplicabilidad de lo discutido hasta ahora. Este Eje está formado por tres Programas: Indisciplinar la Escuela; KM2: Arte y Comunidad; Comunicación $360^{\circ}$, y cada uno de los programas tiene un conjunto de medidas. También por la misma razón, es decir, una mayor aplicabilidad a la temática reflejada en este artículo, destacamos el Programa Escolar Indisciplinario y el Proyecto Cultural Medida Escolar, como espacios privilegiados para la construcción de estrategias fructíferas en el campo de la educación intercultural.

En este sentido, el Programa pretende reforzar la identidad de cada grupo de escuelas considerando su contexto territorial, social y cultural; y articular la escuela, el currículo, los contenidos, el territorio, la comunidad, el patrimonio y la cultura local, promoviendo, a través del Proyecto Escuela 
Cultural, una escuela conectada, abierta y creativa, en línea con los Objetivos de Desarrollo Sostenible de la ONU. Objetivos de Educación de la OCDE 2030, Carta del Consejo de Europa sobre Educación para la Ciudadanía Democrática y los Derechos Humanos, entre muchos otros, en el área de la interculturalidad y la educación intercultural (PNA, 2019).

Así, el Plan Nacional de las Artes, con las artes como principal herramienta y la relación entre arte y educación como primer objetivo, busca y promueve la educación intercultural, donde los proyectos culturales desarrollados en los grupos escolares juegan un papel fundamental en la preparación e implementación cultural de programas en los grupos para el disfrute y la producción, integrando la diversidad de manifestaciones y lenguajes artísticos, en formatos transdisciplinares.

En definitiva, el arte es una herramienta poderosa en la construcción de relaciones educativas interculturales, donde las comunidades educativas amplían los significados de entenderse a sí mismas y a los demás, contribuyendo a sociedades más equitativas y justas. Con esta idea como marco, el Plan Nacional de las Artes, recientemente implementado en Portugal, refuerza la importancia de la interculturalidad, de la educación intercultural, al tiempo que presenta las artes como una forma privilegiada de operacionalizar las estrategias de enseñanza-aprendizaje que promueven el conocimiento, la integración y el encuentro de culturas.

\section{Conclusión}

Las sociedades contemporáneas son más que lugares de pluralismo, diversidad cultural y multiculturalismo: son espacios cada vez más buscados para la convivencia armónica y la interculturalidad. En realidad, hoy se aboga por un nuevo paradigma, que afirma no solo lo diferente, sino también lo común, y que busca crear un marco de convivencia en el que ningún grupo sea discriminado por aspectos diferenciadores. Este cambio de paradigma se refleja en los ámbitos sociales más variados, a saber, en el ámbito educativo.

Así siendo, es importante resaltar que los contextos escolares son lugares privilegiados para la construcción de la interculturalidad y los procesos de educación intercultural. Y porque la educación intercultural es poder partir desde el punto de vista del otro, para construir espacios de aprendizaje formados por estructuras de relaciones que se crean y recrean en los inter lugares de la diversidad cultural, las artes y los lenguajes artísticos emergen como herramientas útiles para el aprendizaje y el desarrollo personal y colectivo.

La Escuela reconoce en el arte y los lenguajes artísticos la capacidad de enriquecer modelos pedagógicos para nuevas formas de aprendizaje y la construcción de relaciones sociales, en la búsqueda de una experiencia 
armónica y en el entendimiento intercultural en el aula, en la escuela y en la comunidad.

Así, y en la construcción de contextos escolares cada vez más inclusivos, surge el Plan Nacional de las Artes, que reitera la importancia de las artes en la promoción de prácticas educativas más igualitarias, ricas e innovadoras. Por ello, el Plan se destaca como una importante estrategia de actuación en la lucha contra la exclusión social y en la adaptación de la educación a la diversidad del alumnado y del resto de la comunidad escolar.

Debido a las limitaciones impuestas por el contexto pandémico, las escuelas, en la búsqueda de una operacionalización apresurada y sin precedentes de su misión educativa a través de nuevos modos de enseñanzaaprendizaje, dejaron en suspenso el Plan Nacional de las Artes. De hecho, con la pandemia, y con todas las restricciones asociadas, gran parte de lo que ya se había determinado en las escuelas bajo este Plan tuvo que ser suspendido. Sin embargo, con la conciencia de que hoy se vive una nueva normalidad, más que nunca, el Plan Nacional de las Artes es importante.

Aunque falten algunos ajustes y adaptaciones, Portugal debe esforzarse por mantener el rumbo definido e implementar el Plan en las escuelas portuguesas. Ahora, precisamente por todas las limitaciones actuales, las artes se revelan como de gran importancia para potenciar la acción de los programas y redes de educación intercultural y de los contactos y relaciones entre todos en la escuela. Hoy más que nunca, las escuelas necesitan reforzar su acción en la articulación, no solo entre currículo, contenidos, territorio, sino también, involucrar identidades colectivas e individuales. No solo durante el contexto de la pandemia, sino también en la pospandémica, el PNA, es decir, a través del Proyecto Escuela Cultural, será un territorio fértil en instrumentos y estrategias para ayudar a toda la comunidad educativa a (re) encontrarse en una perspectiva de conocimiento mutuo e integración, respetando el derecho de cada persona a su propia identidad, en una progresión en el respeto a los Derechos Humanos.

\section{References:}

1. Almeida, V. (2010). O mediador sócio-cultural em contexto escolarContributos para a compreensão da sua função social. Mangualde: Edições Pedago.

2. Branco, J. (2021). Da integração dos saberes...para a integração social.... In N. Ferreira \& C. Nunes (Org.), Diversidades, Educação e Inclusão (pp. 313-331). Lisboa: Escola Superior de Educação, Instituto Politécnico de Lisboa.

3. Candau, V. (2008). Direitos humanos, educação e interculturalidade:

4. as tensões entre igualdade e diferença. Revista Brasileira de Educação, 13 (37), 45-56. Disponível em 
https://www.scielo.br/pdf/rbedu/v13n37/05.pdf

5. Cornejo, J. (2012). Educación, interculturalidad y ciudadanía. Educar em Revista, 43, 239-254. https://doi.org/10.1590/S010440602012000100016

6. Fortuna, C. (coord.) (2014). Cultura, Formação e Cidadania Relatório Final. Lisboa: Gabinete de Estratégia, Planeamento e Avaliação Culturais - Secretaria de Estado da Cultura.

7. Giménez, C. (2010). Interculturalidade e Mediação. Cadernos de Apoio à Formação. Lisboa: ACIDI, I.P.. Disponível em https://www.acm.gov.pt/documents/10181/233158/4.+Interculturalid ade+e+Media\%C3\%A7\%C3\%A3o.pdf/2c32448e-acee-4788-90f1483e101f2ff9?version $=1.0$

8. Pierucci, A. (1999). Ciladas da diferença. São Paulo: Editora 34.

9. Plano Nacional das Artes - Uma estratégia, Um manifesto 2019-2024 (PNA) (2019). Lisboa: Ministério da Cultura e Ministério da Educação. Disponível em https://www.dge.mec.pt/sites/default/files/Projetos/PNA/Documentos lestrategia_do_plano_nacional_das_artes_2019-2024.pdf

10. Rede de Escolas para a Educação Intercultural [REEI] (2017). Termos de Referência. Lisboa: Direção-Geral de Educação. Disponível em https://www.dge.mec.pt/sites/default/files/ECidadania/Educacao_Inte rcultural/documentos/reei_termos_de_referencia.pdf

11. Sousa Santos, B. (2003). Reconhecer para libertar: os caminhos do cosmopolitanismo multicultural. Rio de Janeiro: Civilização Brasileira.

12. Sousa, J. (2020). Emoções, Artes e Intervenção: os elementos estruturantes da Animação Artística. In J. Sousa, Santos, M.J. \& Lopes, M.S.P. (Org.). Emoções, Artes e Intervenção (pp. 6-15). Leiria: Escola Superior de Educação e Ciências Sociais - Politécnico de Leiria, CICS.NOVA.IPLeiria, Centro de Estudos em Educação e Inovação e Universidade FEEVALE.

13. Sousa, J., Lopes, M.S.P. \& Kowalski, M. (2020). Educação Artística: Intervenção e Investigação em Contextos Escolares. Leiria: Escola Superior de Educação e Ciências Sociais - Politécnico de Leiria. Disponível em https://www.ipleiria.pt/esecs/wpcontent/uploads/sites/15/2020/06/Liv ro_Educacao_Artistica.pdf

14. Stoer, S. \& Cortesão, L. (1999). Levantando a Pedra: da pedagogia inter/multicultural às políticas educativas numa época de transnacionalização. Porto: Edições Afrontamento.

15. UNESCO (2005). Convenção sobre a Proteção e Promoção da Diversidade das Expressões Culturais. Adotada pela 33. ${ }^{a}$ sessão da 
Conferência Geral da UNESCO, em 20 de outubro de 2005 - aprovada pela Resolução da Assembleia da República n. ${ }^{\circ}$ 10-A/2007, Diário da República n. ${ }^{\circ} 54$ - I Série, de 16 de março de 2007.

16. UNESCO (2006). Guidelines on intercultural education. Disponível em http://unesdoc.unesco.org/images/0014/001478/147878e.pdf. 